\title{
Nicht förderungswürdig
}

Weshalb die Evaluation der Exzellenzinitiative gegen deren Fortsetzung spricht

\author{
Tilman Reitz \\ Angela Graf \\ Christina Möller
}

Die Evaluationen, die seit den späten 1990er-Jahren in der öffentlichen Verwaltung um sich greifen, helfen bekanntlich Entscheidungen zu rechtfertigen. Zumal wenn knappes Geld verteilt werden soll, ist eine Empfehlung durch Expert_innen und Angehörige des Feldes hochwillkommen. Die vom Bund initiierte und von den Ländern mitgetragene Exzellenzinitiative nutzt das Instrument der Evaluation inzwischen in doppelter Weise. Erstens werden im Wettbewerb um Exzellenzmittel selbst Einrichtungen, Projekte und ,Visionen' evaluiert. In Anlehnung an auch sonst üblich gewordene PeerReview-Verfahren wählen Fachjurys jene aus, die aufgrund zugeschriebener Qualität die Gelder erhalten sollen. Die Evaluation und Entscheidung erfolgt dabei weniger auf Grundlage bisher erbrachter Leistungen als auf Grundlage der Anträge, also der überzeugenden Darstellung versprochener zukünftiger Leistungen.[1] Inwiefern damit tatsächlich wissenschaftliche Exzellenz beurteilt oder vielmehr erst hergestellt wird, wurde seit Beginn der Exzellenzinitiative kontrovers diskutiert (zum Beispiel Münch 2007a; 2007b, Hartmann 2006; 2010, Kühl 2016). In jedem Fall wird durch dieses Instrument eine sonst hochstrittige Bündelung von Mitteln im öffentlichen Diskurs an wissenschaftliche Standards gekoppelt. Zweitens wurde mit der Evaluation der Exzellenzinitiative von den Geldgeber_innen nun zusätzlich eine Art Meta-Evaluation installiert, die das Programm selbst bewerten soll. Der Legitimationszweck lag hier klar auf der Hand, wurde aber wenig geschickt verfolgt: Die Ergebnisse waren insgesamt absehbar und werden im Detail von den Entscheidungsträger_innen nur höchst selektiv aufgenommen.

Mit der Evaluation beauftragt und um Empfehlung gebeten wurde die sogenannte Internationale Expertenkommission Exzellenzinitiative(IEKE), zu deren Konstitution im Kommissionsbericht nur wenig vermerkt ist. Der Vorsitzende Dieter Imboden, ein erklärter Anhänger der Exzellenzinitiative, wurde durch die Gemeinsame Wissenschaftskonferenz (GWK) berufen, alle weiteren Kommissionsmitglieder wurden von ihm vorgeschlagen und durch die GWK lediglich bestätigt (IEKE 2016: 8). Der Physiker Imboden, den seine langjährigen Leitungsfunktionen im Schweizer Nationalfonds und im Österreichischen Wissenschaftsfonds für die Aufgabe qualifizieren, hat sich für die Evaluation 
Kolleg_innen gesucht, die ähnlich wie er Wissenschaft und Verwaltungsämter verbinden. Die staatliche Geldvergabe durch Drittmittelwettbewerb ist für viele von ihnen selbstverständliche Praxis, kein skeptisch zu prüfender Vorgang.[2] Zudem fällt auf, dass das Fächerspektrum der zehn Kommissionsmitglieder begrenzt ist: Medizin, Jura, (mehrfach) Physik, Informatik, Wirtschaftsund Geschichtswissenschaft - weitere Geisteswissenschaften fehlen ebenso wie Wissenschaften, die auf die Analyse sozialer Prozesse und evaluativer Methoden spezialisiert sind (Soziologie, Psychologie, Erziehungswissenschaft). Daher liegt es nahe, dass bestimmte Formen von Kritik und Selbstreflexion in dem Bericht randständig bleiben. Die Zusammensetzung der Kommission stellt in gewissem Maß eine Vorentscheidung über ihre sachliche Haltung dar. Im Evaluationsbericht spricht sich die Kommission, wie zu erwarten war, für die Fortsetzung der Exzellenzinitiative aus und bietet ihr somit die erwünschte Legitimationsgrundlage.

Schaut man sich den im Januar veröffentlichten Bericht der ImbodenKommission genauer an, kann man trotz dieses positiven Votums erhebliche Zweifel entwickeln, ob eine Verstetigung der Exzellenzinitiative sinnvoll ist: Die Kommission beleuchtet mehr ungelöste Probleme als klare Erfolge der Initiative, und ihre Argumente für eine ausgebaute Spitze sind ausgesprochen angreifbar. Diese Zweifel sollen hier ausgeführt werden. Selbst wenn es zu spät sein sollte, mit unserer Evaluation der Evaluation Entscheidungsbefugte zur Besinnung zu bringen, sammeln wir so rationale Erwägungen für bessere politische Zeiten.

Vieles ist bereits gesagt beziehungsweise geschrieben worden. Seit Beginn der Exzellenzinitiative 2005 wurden grundlegende Argumente gegen die konkurrenzförmige Hierarchisierung der deutschen Hochschullandschaft vorgebracht, die durch sie vorangetrieben wurde. Von der Eliten- und Hochschulsoziologie (z. B. Hartmann 2005; 2015, Münch 2007a, Kühl 2016) bis in die Rechts-, Geistes- und Kulturwissenschaften (z. B. Möllers 2009, Schwindt 2013) und auch in den Naturwissenschaften (z. B. Brembs/Brennicke 2015, Brembs 2016) gibt es kritische Stimmen. Auf diese Argumente werden wir in unserer Auseinandersetzung mit dem Imboden-Bericht wiederholt zurückkommen.

Im Kommissionsbericht selbst wird keiner der genannten Texte erwähnt. Die fehlende Auseinandersetzung mit grundsätzlicher Kritik kann auch als Unklarheit über den Zweck der Evaluation gedeutet werden. Während die Kommission ihre Aufgabe wohl darin sah, die Exzellenzinitiative an ihren selbst gesetzten Zielen zu messen, also eine summative Evaluation vorzulegen, behandeln die politisch Zuständigen den Bericht als Entscheidungsgrundlage, also als formative Evaluation zur Fortführung und Gestaltung eines umstrittenen Programms. In diesem zweiten Kontext wäre eine stärkere Reflexion der möglichen oder wahrscheinlichen (nicht intendierten) Langzeitfolgen der Exzellenzinitiative obligatorisch gewesen.

\section{Die Dauerbaustellen im deutschen Hochschulsystem: wovon die Exzellenzinitiative ablenkt}

Die Evaluationskommission hat trotz ihres positiven Gesamturteils keinen Jubelbericht geschrieben. Sie benennt massive Probleme des deutschen 
Hochschulsystems, zu deren Lösung die Exzellenzinitiative großenteils keinen Beitrag leistet oder die sie noch verschärft. Die „Baustellen“, die der Bericht als besonders dringlich darstellt, sollen in den folgenden Abschnitten genauer betrachtet werden, bevor wir blinde Flecken des Berichts benennen und zu unserer abschließenden Einschätzung kommen.

Im Überblick lassen sich die von der Kommission benannten „Baustellen“ in zwei Hauptrubriken einteilen: Zum einen werden Probleme angeführt, die erst im Kontext von Exzellenzbedürfnissen als solche erscheinen, wie die mutmaßlich zu schwach ausgeprägte vertikale Differenzierung zwischen den Universitäten oder angeblich zu schwache Hochschulleitungen. Zum anderen werden grundlegende Strukturprobleme eindrücklich belegt: Die deutschen Hochschulen sind im internationalen Vergleich und in zunehmendem Maß unterfinanziert; [3] entsprechend hat die Betreuungsbelastung der Lehrenden nach einer kurzen Verbesserung wieder zugenommen und ist heute höher als 2003 (IEKE 2016: 14, 21); schließlich wird diese Last immer umfassender auf einen prekär beschäftigten wissenschaftlichen Nachwuchs abgewälzt (ebd.: $26 \mathrm{f}$ ).

Was leistet die Exzellenzinitiative, um diese Strukturprobleme zu bewältigen? Die offizielle Antwort des Kommissionsberichts lautet: nichts. Das in die Exzellenzförderung investierte Geld ist verglichen mit den Budgets der Stanford University, der University of Oxford oder der ETH Zürich ein Tropfen auf den heißen Stein[4]; die Betreuungsrelationen werden sich weiter verschlechtern, wenn ,exzellente ${ }^{\varsigma}$ Forschende von der Lehre freigestellt werden; die Zahl der zeitlich befristeten Nachwuchs-Projektstellen hat durch die Exzellenzinitiative (und ihre Vorbildwirkung) weiter zugenommen.

Zwischen den Zeilen lässt sich allerdings doch eine Perspektivänderung ablesen: Wenn es den meisten Hochschulen unverantwortbar schlecht geht, könnte man bei einigen eine Ausnahme machen und bei allen anderen die Ansprüche senken. Man muss dazu nur egalitäre Ansichten aufgeben: „Obwohl [bisher] nicht nachweisbar ist, dass sich das deutsche Universitätssystem durch die Exzellenzinitiative stärker ausdifferenziert hätte, wurde durch sie im Zuge der öffentlichen Diskussion zumindest die ,alle-sind-gleichIllusion“ begraben." (IEKE 2016: 19) Sätze wie dieser betreffen nicht nur die Förderung bestimmter Schwerpunkte an bestimmten Standorten. Sie verlangen auch eine größere ,Differenzierung' zwischen ,Oben“ und ,Unten', zwischen ,Elite‘ und ,Masse‘. Die offensichtlichen Strukturprobleme des deutschen Wissenschafts- und Hochschulsystems werden dadurch nicht nur marginalisiert, sondern auch legitimiert - und für die Verlierer_innen im Exzellenzwettbewerb noch verschärft.

\section{Vertikale Differenzierung: das Matthäus-Prinzip in offener und verschleierter Form}

Der Imboden-Bericht hat in vielen Punkten den Vorzug der Klarheit. Als zentrales Ziel der Exzellenzinitiative weist er die „vertikale Differenzierung“[5] des deutschen Hochschulsystems aus, also eine Schaffung von Hierarchien zwischen den Hochschulen und bei begrenzten Mitteln eine Umverteilung von unten nach oben. Das dürfte die Erwartung einschließen, dass die Exzellenz-Universitäten symbolische und strukturelle Strahlkraft entwickeln, 
die weit über die finanzielle Subventionierung im Volumen von mehr als 500 Millionen Euro pro Jahr hinausgehen. Dieses Ziel wurde lange geleugnet: Der Eliteforscher Michael Hartmann hatte es bei der zweiten Runde der Exzellenzinitiative noch entlarvend herausgestellt (Hartmann 2015), und noch in den Diskussionen über den wünschenswerten Zuschnitt der aktuellen Fortsetzung wurde häufiger eine ,Exzellenz in der Breite beschworen.

Die Klarheit, die von der Kommission geschaffen wurde, hat die Hochschulpolitik dankbar aufgenommen - allerdings nur bis zu einem bestimmten Punkt. Sie will nämlich nicht durch die Vergaberegeln sichtbar machen, dass ganz im Sinne des Matthäus-Effekts (Merton 1985; 2010) vorrangig denen gegeben wird, die schon haben.[6] Der Vorschlag der Kommission, einigen wenigen Universitäten automatisch beziehungsweise aufgrund von Kennzahlen eine jährliche Exzellenzprämie von zehn bis fünfzehn Millionen Euro zu zahlen (IEKE 2016: 43 ff.)[7], hat sich nicht durchgesetzt. Auch wenn schon jetzt absehbar ist, welche Einrichtungen im Wettbewerb die besten Chancen haben, und obwohl gerade bei den vorgesehenen acht bis elf Exzellenz-Universitäten nicht viele Überraschungen zu erwarten sind, soll die Hierarchie vollständig durch Anträge und deren Bewertung hergestellt werden. Der Rechtfertigungsvorteil liegt auf der Hand: Die als Beste Beglaubigten setzen sich durch, und auch die faktischen Mittelkürzungen bei der Nicht-Spitze erscheinen als leistungsgerechtes Ergebnis.

Die fatalen Nebenfolgen der Wettbewerbe, von der bloßen Antragsarbeit bis zu Anreizen für die rhetorische oder betrügerische Aufpolierung von Forschungsergebnissen, werden noch zu diskutieren sein. Doch auch das Hauptziel der Vertikalisierung ist ein Problem, da es vor allem Verlierer_innen hervorbringen wird.[8] Die traditionelle Stärke des deutschen Hochschulsystems bestand bislang darin, dass an prinzipiell jedem Standort fast jedes Niveau von Forschung möglich ist - auch international sichtbare Spitzenforschung. Das Vorhaben, diese Forschung an wenigen Standorten zu bündeln, verspricht wenig Gewinn, aber klare Verluste. Die durch den Exzellenzwettbewerb nebenher auf Dauer gestellte und legitimierte Mittelkürzung an den nicht erfolgreichen Hochschulen wird die Arbeits- und Forschungsbedingungen dort weiter verschlechtern. Sie werden dauerhaft ihre Attraktivität für internationale Studierende und Gastforscher_innen verlieren und ihre Bedeutung wird tendenziell auf eine rein regionale Ebene absinken. Zugleich droht das Postulat der Einheit von Forschung und Lehre zunehmend aufgegeben zu werden. Die Exzellenz-Universitäten und Standorte von Exzellenzclustern werden durch Bereitstellung entsprechender finanzieller Ressourcen verstärkt zu Forschungsuniversitäten aus- beziehungsweise umgebaut, während die restlichen Hochschulen tendenziell zu Ausbildungsanstalten degradiert werden. Diese Stoßrichtung wird unter anderem durch Deputatsreduktionen beziehungsweise die Befreiung ,exzellenter Forschender von der Lehre deutlich, die im Gegenzug durch erhöhte Lehrbelastung aller anderen erkauft wird.

\section{Prekäre Beschäftigung des Nachwuchses: erkannte Probleme, vermiedene Lösungen}

Wie die Imboden-Kommission zu Recht feststellt, wurde die miserable Beschäftigungssituation des wissenschaftlichen ,Nachwuchses ${ }^{6}$ in 
Deutschland durch die Exzellenzinitiative weiter verschlechtert. Die Zahl der (häufig sehr kurzzeitig) befristeten Stellen unterhalb der Professur nimmt kontinuierlich zu, Dauerstellen bleiben die Ausnahme. Besonders durch die explosive Zunahme von Projektstellen verschärft sich die „Flaschenhalsproblematik“, also das Problem, dass „einer hohen Zahl qualifizierter und befristet angestellter Nachwuchswissenschaftler_innen eine geringe Zahl von Professuren bzw. sonstiger Dauerstellen gegenübersteht“ (IEKE 2016: 27). Die Kommission kommentiert dies sehr deutlich:

„Die Situation ist insofern nicht ganz frei von Zynismus, als die Universitäten immens davon profitieren, dass sich eine große Zahl junger Menschen darauf einlässt - in der Hoffnung auf eine akademische Karriere - die produktivsten Jahre ihres Lebens auf schlecht bezahlten und befristeten Post-Doc-Stellen zu verbringen.“ (ebd.: 26)[9]

Die Kommission empfiehlt daher, das Ziel der Nachwuchsförderung und das Instrument der Graduiertenschulen ganz aus der Initiative zu streichen (IEKE 2016: 35 f., 39 f.); die Ministerien haben den Streichungsvorschlag übernommen und planen zudem, zukünftig eher Tenure-Track-Stellen statt bloßer Projektstellen zu schaffen. Derart lässt sich vielleicht der schon entstandene und durch die Exzellenzinitiative vergrößerte strukturelle Schaden eindämmen, allerdings um den Preis, dass sich die auf Dauer angelegte Förderung bei einer kleinen Gruppe auserwählter Nachwuchsforschender bündelt (die Rede ist von circa 1.0oo Stellen), während die schlechte Lage aller anderen (die derzeit auf etwa 145.000 befristeten Mittelbaustellen arbeiten[10]) weiterhin besteht und zudem durch den Wettbewerb legitimiert wird.

\section{Ein blinder Fleck des Berichts: verschärfte soziale Ungleichheit}

Darüber hinaus ist zu erwarten, dass sich ein Effekt verschärft, den die Imboden-Kommission nicht anspricht, nämlich erneut zunehmende soziale Ungleichheiten beziehungsweise Herkunftsvorteile im Wissenschaftsbetrieb. Für eine erfolgreiche Positionierung in der Wissenschaft war die soziale Herkunft auch bisher schon von großer Bedeutung (Hartmann 2002, Möller 2015, Graf 2015). Zahlreiche Studien zeigen, dass Nachwuchswissenschaftler_innen aus bildungsaffinen und kapitalstarken Elternhäusern bereits Kenntnisse über erfolgreiche Karrierestrategien und wissenschaftliche Spielregeln mitbringen, die für eine frühe soziale Positionierung im akademischen Feld hilfreich sind, während soziale Aufsteiger_innen sich diese erst noch aneignen müssen (Hasenjürgen 1996, Lange-Vester/Teiwes-Kügler 2013). Prekäre und risikoreiche Beschäftigungsbedingungen verstärken diese Ungleichheiten zusätzlich. Die Karriereunsicherheit stellt gerade für soziale Aufsteiger_innen eine enorme Hürde dar. Dagegen sind Personen aus sozial und ökonomisch privilegierten Familien prinzipiell eher in der Lage, sich auf eine risikoreiche Karriere einzulassen, da sie über ein familiäres Sicherheitsnetz verfügen. Auch die Imboden-Kommission sieht eine deutliche Gefahr, 
dass es „nicht die ,besten Köpfe“ sind, die sich auf dieses Vabanquespiel einlassen“ (IEKE 2016: 26). Sie bezieht sich damit allerdings nur auf Geschlechterungleichheiten, nicht auf soziale Selektivität. Dass diese Selektivität zunimmt, wenn Wettbewerbe inszeniert und den Erfolgreichen bereits früh privilegierte Positionen zugeteilt werden, zeigt sich auch in neueren Tendenzen sozialer Schließung in der Professor_innenschaft und besonders bei den Juniorprofessuren (Möller 2015). Unter diesem Gesichtspunkt wäre selbst der Ausbau von ,exzellenten` Tenure-TrackStellen ein bestenfalls fragwürdiger Gewinn.

Hinsichtlich der bereits erwähnten Geschlechtergerechtigkeit - dem einzigen sozialen Faktor, den die Imboden-Kommission thematisiert und problematisiert (IEKE 2016: 27) - fehlen überzeugende Maßnahmen. Trotz Gleichstellungsbemühungen sind Frauen in Führungspositionen der Exzellenzeinrichtungen Ausnahmen geblieben (Engels et al. 2015) und auch im wissenschaftlichen ,Nachwuchs“ scheinen sie weiterhin Verliererinnen in verschärften Wettbewerbssituationen zu sein (Funken et al. 2015). Der von der Kommission vorgeschlagene Wegfall der „Zukunftskonzepte“ (IEKE 2016: 43), in denen Gleichstellung stark gemacht wurde, lässt zudem eine erneute Marginalisierung dieser Thematik befürchten.

Die weiter reichenden Auswirkungen der Exzellenzinitiative auf soziale Ungleichheiten sind im Imboden-Bericht wie in vielen anderen Debattenbeiträgen nicht einmal am Horizont sichtbar. Es ist anzunehmen, dass die Exzellenz-Universitäten zu Elitebildungsstätten im Wortsinn werden. Der Blick auf stark vertikal differenzierte Hochschulsysteme beziehungsweise auf Länder, in denen Elitehochschulen Tradition haben, macht deutlich, dass die soziale Herkunft für die Aufnahme und das erfolgreiche Studium an diesen Institutionen enorme Bedeutung hat. Nicht nur die hohen Studiengebühren, die etwa von Eliteuniversitäten in den USA erhoben werden, sondern auch die aufwändigen Selektions- und Aufnahmeverfahren wie an den Grandes Écoles in Frankreich, bei denen die soziale Herkunft vermittelt über den Habitus (und die Kosten für Vorbereitungskurse) eine zentrale Rolle spielt, tragen zur sozial exklusiven Zusammensetzung der dort Studierenden bei[11]; auf dem Arbeitsmarkt zahlt sich der Abschluss dann in hohen Einstiegsgehältern und im Zugang zu Führungspositionen aus (Rivera 2015, Binder et al. 2016). Ansatzweise lässt sich auch in Deutschland bereits eine erhöhte Attraktivität von Exzellenz-Universitäten für Studierende aus bildungs- und finanzstarken Elternhäusern belegen (Stiftung neue Verantwortung 2011). Alles spricht dafür, dass sich dieser Trend durch die Verstetigung der Exzellenzinitiative fortsetzen wird.[12]

Die symbolische Strahlkraft der Exzellenz-Universitäten dürfte schließlich langfristig dazu führen, dass die Wege an die wissenschaftliche Spitze zunehmend durch wenige Institutionen verlaufen - nicht nur mit sozialstrukturellen, sondern auch mit epistemischen Folgen.[13] Die vertikale Hierarchisierung und soziale Selektivität der Hochschulen befördert zugleich die Deutungshoheit derer, die an den selektiven, ,exzellenten' Einrichtungen forschen. Dies hätte negative Auswirkungen auf die Perspektivenpluralität, die Kritikfähigkeit und Qualität der Wissenschaft als solcher, und würde dem gesetzten Ziel, der Förderung von Spitzenleistungen, geradezu entgegenwirken. 


\section{Governance oder starke Hochschulleitungen: ein Vorbild ohne Argumente}

Die Imboden-Kommission sieht die Probleme ihrem Auftrag entsprechend in der zu wenig herausgehobenen Spitze. Das gilt auch für hochschulinterne Hierarchien: Die Kommission wünscht sich starke Hochschulleitungen in Deutschland. An die Stelle der Analysen und begründeten Argumente treten dabei mehrheitlich Meinungsbekundungen beziehungsweise summarische, unüberprüfbare Urteile:

„Handlungsfähigkeit, Autonomie und Wettbewerb haben sich als die wichtigsten Faktoren für den Erfolg einer Universität erwiesen [...]. Im Inneren sollte die Entscheidungskompetenz dort angesiedelt sein, wo auch für Folgen eingestanden werden kann, d. h. bei den zentralen (Präsidium/Rektorat) oder dezentralen (Dekanat) Leitungsebenen. [...] Im Außenverhältnis bedeutet Autonomie, dass die Freiheit von Forschung und Lehre die Freiheit einschließt, Prioritäten zu setzen."(IEKE 2016: 20)

Auch „Posterioritäten“, das heißt zentral verfügte Kürzungen in einigen Bereichen, sollen durch „starke Governance“ (ebd.: 38) möglich werden. Das kann man anstreben oder auch nicht; der einzige Ansatz für eine Begründung besteht im Verweis auf „internationale Spitzenuniversitäten“, die „eine starke interne Governance“ haben und „,von staatlichen Eingriffen weitgehend abgeschirmt" (ebd.: 21) sind. Dass diese Universitäten ihren Erfolg vielleicht eher den andernorts herausgestellten Riesenbudgets verdanken, bleibt unreflektiert.

Überlegt man genauer, welche Leitungsentscheidungen im deutschen Exzellenz-Biotop möglich sind, verliert eine starke Führung deutlich an Plausibilität: Der Effekt besteht hier wesentlich darin, dass Entscheidungsträger_innen im Präsidium oder Dekanat den mit dem Forschungsstand besser Vertrauten in den Instituten und Arbeitsbereichen vorschreiben, welche Vorhaben sie vorrangig zu verfolgen haben und mit wem sie zusammenarbeiten sollen. Organisationale Zielsetzungen schieben sich so zunehmend vor wissenschaftlich anvisierte Themensetzungen und Forschungsprogramme; Forschungsentscheidungen werden immer mehr zu strategischen Entscheidungen, die in komplexen Aushandlungsprozessen zwischen verschiedenen Interessensgruppen gefällt werden (Aliets/Lettkemann 2012), was eine weitere Reduktion der wissenschaftlichen Pluralität sowie gewagter Forschungsvorhaben abseits des Mainstreams befürchten lässt. Kritisch ausgerichtete Arbeitsbereiche, die sich bisher immerhin eigenständig in die Drittmittelkonkurrenz wagen konnten, müssen sich in diesem Kontext zusätzlichen ideologischen Vorgaben anpassen. Exzellenz-Universitäten, deren Präsidien mit weitreichenden Machtbefugnissen ausgestattet sind, und solche, die sich für den Exzellenzwettbewerb rüsten, könnten den bereits bestehenden Drift zum Mainstream noch einmal deutlich verstärken.

\section{Die Grundsatzfrage: Sinn und Funktion wissenschaftlicher Antrags- und Statuswettbewerbe}

Ein letzter Punkt sollte mit und ohne den Imboden-Bericht klar sein: Der anhaltende Trend zum Prestige-Wettbewerb und zu Quasi-Märkten im 
Hochschulsektor bedroht die Substanz, die Attraktivität und die kritischen Potenziale wissenschaftlicher Arbeit. Statt für eine solide Grundfinanzierung zu sorgen, die gute Lehre gewährleistet und Freiraum für Forschung lässt, treibt die deutsche Wissenschaftspolitik die Forschenden in eine Dauerkonkurrenz um die weiterhin staatlich bereitgestellten knappen Mittel. Sie verstärkt damit eine Fassadenkultur (bei der der Antragserfolg wichtiger wird als die Durchführung), eine Mainstream-Orientierung (infolge derer man sich schon bei der Projektplanung der vermuteten Meinung der anderen anpasst, sowie mit unorthodoxen Denkrichtungen wenig Erfolgschancen hat und oft gleich von Anträgen absieht) und prekäre Arbeitsverhältnisse in der Wissenschaft (in denen diskontinuierliche Beschäftigung durch Bindung an Forschungsprojekte normal wird oder bleibt).

Grundsätzlich verkehrt das Antragswesen die Zweck-Mittel-Relation im Forschungsbetrieb (Kühl 2016). Statt Mittel zu beantragen, wenn Forschungsvorhaben kostspielig sind - weil man zum Beispiel Apparate für Experimente oder Personal für Interviews braucht -, muss man im deutschen Wissenschaftssystem in Drittmittelwettbewerben erfolgreich sein, um seine Beschäftigung zu sichern und seinen Marktwert zu steigern. Die Zeit, in der man forschen könnte, und die kollegialen Kooperationen, die man vielleicht gerne aufbauen würde, werden so von einem Spiel aufgezehrt, dessen Mitspieler_innen unter den Forschenden bevorzugt ,Beutegemeinschaften“ heißen. Neben den dysfunktionalen Zügen, die dieses Spiel hat, zerstört es zunehmend auch die intrinsische Motivation für die wissenschaftliche Tätigkeit.

Den Forschenden und Lehrenden sind die meisten Schwächen der Exzellenzinitiative geläufig; sogar eine mehrheitliche Ablehnung des Programms ist belegt.[14] Sie beugen sich allerdings praktisch (notgedrungen) dem Wettbewerbs- und Erfolgsdruck der Kolleg_innen, Vorgesetzten, Fakultäten, Hochschulleitungen, Landesregierungen und Bundesministerien und verinnerlichen zunehmend die Logik, Antragserfolge mit wissenschaftlicher Qualität gleichzusetzen. Daher wird es ihnen wohl wieder so ergehen wie in den ersten beiden Runden: Wieder werden sie monatelang Anträge für den Geld- und Prestigewettbewerb schreiben, wieder wird trotz voraussehbarer Grundmuster mit Spannung erwartet werden, wer genau im Wettbewerb gewinnt und verliert, wieder wird der Prozess von den grundsätzlichen Strukturproblemen des deutschen Hochschulsystems ablenken, die auch der Bericht der Imboden-Kommission feststellt. Wissenschaftlich arbeitenden und denkenden Menschen, die auch die eigene Lage analysieren und vernünftige Schlussfolgerungen ziehen wollen, bleibt nur die Möglichkeit, sich kritisch gegen die Verstetigung der Exzellenzinitiative zu positionieren und einen Politikwechsel zu fordern. Selbst innerhalb der vorherrschenden Rechtfertigungslogik bestätigt sich diese Einschätzung: Da die Exzellenzinitiative ihrer kritisch evaluierten eigenen Evaluation nicht standhält, ist sie nicht weiter förderungswürdig. 


\section{Endnoten}

[1] Zur Konstruktion wissenschaftlicher Leistung siehe beispielsweise Beaufaÿs/Krais 2007, Graf 2015: $61 \mathrm{ff}$.

[2] Zwei weitere Mitglieder der Kommission (Georg Kratky und Michael Schneideregger) waren führend im Österreichischen Wissenschaftsfonds und im Schweizer Nationalfonds tätig. Für alle Mitglieder, außer für Imboden selbst, sind hoch dotierte Forschungspreise und Projektförderungen dokumentiert (IEKE 2016: 62f).

[3] Die Ausgaben pro Studienplatz sind in Deutschland höchst bescheiden und im Zeitraum von 2003 bis 2013 (v. a. aufgrund steigender Studierendenzahlen) gesunken (IEKE 2016: 12 f). Zur internationalen Einordnung vergleicht der Bericht zwar nur selektiv US-amerikanische, englische und Schweizer ,Spitzenuniversitäten' mit solchen, die es in Deutschland werden könnten (Konstanz, RWTH Aachen; ebd.: 15), doch auch ein struktureller Vergleich mit Studiengebühren erhebenden Ländern wie den USA oder mit den skandinavischen Sozialstaaten kann Defizite belegen (Schulze-Cleven/Olsen 2016, i. E.).

[4] Das jährliche Budget der Exzellenzinitiative beträgt weniger als ein Siebtel des Jahresbudgets der Standford University und deutlich weniger als die Hälfte der Budgets der University of Cambridge oder der ETH Zürich (IEKE 2016: 15).

[5] Zur Erläuterung des Begriffs heißt es, dass „sich Universitäten [...] erheblich in Bezug auf Umfang und Qualität ihrer Forschungsleistung“ unterschieden; es folgt die Feststellung: „Die Exzellenzinitiative ist in ihrem Kern auf vertikale Differenzierung zur Formierung besonders forschungsstarker Universitäten (,World Class Universities') ausgerichtet.“(IEKE 2016: 17f.)

[6] Mit dem Matthäus-Effekt nimmt Merton Bezug auf eine Bibelstelle im Matthäusevangelium. Dort heißt es: „Denn wer da hat, dem wird gegeben werden, dass er Fülle habe; wer aber nicht hat, von dem wird auch genommen, was er hat." (Mt 25,29 LUT) Er verweist damit auf die ungleiche Kumulation von Chancen, die in der Konsequenz zu einer Polarisierung der Opportunitätsstruktur führt.

[7] Bemerkenswert ist dabei nicht allein die Kritik an bloß versprochenen Ergebnissen, sondern auch der offensive Vorschlag, eine Kennzahlensteuerung nach britischem Muster im deutschen Wissenschaftssystem zu etablieren. Zwischen diesen beiden Möglichkeiten bewegt sich eine auf Wettbewerb setzende Hochschulpolitik.

[8] Auch dieser Mechanismus ist dem Matthäus-Effekt implizit: Mit der Etablierung von Gewinner_innen werden dieVerlierer_innen gleichsam mitproduziert. Michael Hartmann (2015) hat auf den Trend hingewiesen, dass die Hochschulen ohne Exzellenzmittel auch sonst zunehmend schlechtere Erfolgsquoten bei DFG-Anträgen haben.

[9] Vgl. hierzu ausführlicher Rogge 2015. Im Vergleich mit Frankreich, England und den USA liegt Deutschland mit seiner ungezügelten Befristungspraxis mit Abstand vorn (Konsortium Bundesbericht Wissenschaftlicher Nachwuchs 2013: 82).

[10] Da nur ein Bruchteil der Promovierenden dauerhaft im Wissenschaftsbetrieb verbleiben möchte, sollte die Laufzeit der Stellen, die zur Promotion dienen, zumindest der für die Fertigstellung der Qualifikationsarbeit benötigten Zeit entsprechen. Für jene, die sich für eine dauerhafte Wissenschaftslaufbahn entscheiden, müssen hingegen langfristige Perspektiven in Form von Tenure-Track-Optionen und Entfristungen installiert werden.

[11] Vgl. im Überblick zu den USA, Frankreich, Großbritannien und Japan: Hartmann 2005, ausführlich zu den USA: Mettler 2014, Karabel 2005.

[12] Ansatzpunkte finden sich etwa in der zunehmenden Korrelation hoher Ranking-Plätze von Instituten und sozialer Selektivität bei den Studierenden (Weiss et al. 2015).

[13] Neuere Forschungen zur Reputationshierarchie in der Wirtschaftswissenschaft zeigen, dass die Vergrößerung und verbesserte finanzielle Ausstattung von Instituten mit der Erhöhung ihrer Erfolge in der wissenschaftlichen Gemeinschaft und der Gefragtheit ihrer Angehörigen als Expert_innen in der Öffentlichkeit eng zusammenhängen (Maeße 2015).

[14] 2010 ergab eine Umfrage unter 1000 Professor_innen, dass eine klare Mehrheit die Initiative als „,öllig ungeeignet“ oder „eher ungeeignet“ ansah, um den Wissenschaftsstandort Deutschland zu stärken (Böhmer et al. 2011). U.a. gab es eine Petition gegen die Fortsetzung der Exzellenzinitiative, die über 3.ooo Personen unterzeichnet haben, zu großen Teilen aus der Wissenschaft. (https://www.openpetition.de/petition/online/ fuer-gute-forschung-und-lehre-argumente-gegen-die-exzellenzinitiative, letzter Zugriff 11.11.2016) 


\section{Autor_innen}

Tilman Reitz ist Soziologe und Philosoph. Seine Schwerpunkte sind Politische Theorie, Gesellschaftstheorie, Wissenssoziologie und Ästhetik.

tilman.reitz@uni-jena.de

Angela Graf ist Soziologin. Zu ihren Forschungsschwerpunkten zählen Wissenschafts- und Elitesoziologie sowie Soziale Ungleichheit und Sozialstruktur.

angela.graf@tum.de

Christina Möller ist Soziologin mit den Forschungsschwerpunkten Soziale Ungleichheiten und Bildung sowie Hochschul- und Wissenschaftsforschung.

christina.moeller@uni-paderborn.de

\section{Literatur}

Aliets, Enno / Lettkemann, Eric (2012): Hochschulleitung und Forscher: Von wechselseitiger Nichtbeachtung zu wechselseitiger Abhängigkeit. In: Uwe Wilkesmann / Christian C. Schmid (Hg.), Hochschule als Organisation. Wiesbaden: Springer VS, 131-153.

Beaufaÿs, Sandra / Krais, Beate (2007): Wissenschaftliche Leistung, Universalismus und Objektivität. Professionelles Selbstverständnis und die Kategorie Geschlecht im sozialen Feld Wissenschaft. In: Regine Gildemeister / Angelika Wetterer (Hg.), Erosion oder Reproduktion geschlechtlicher Differenzierungen? Widersprüchliche Entwicklungen in professionalisierten Berufsfeldern und Organisationen. Münster: Westfälisches Dampfboot, 76-98.

Binder, Amy J. / Davis, Daniel B. / Bloom, Nick (2016): Career Funneling: How Elite Students Learn to Define and Desire 'Prestigious' Jobs. In: Sociology of Education 89/1, 20-39.

Böhmer, Susan / Neufeld, Jörg / Hinze, Sibylle / Klode, Christian / Hornbostel, Stefan (2011): Forschungsbedingungen von Professorinnen und Professoren an deutschen Universitäten. Bonn: iFQ-Working Paper 8.

Brembs, Björn (2016): Data show "excellence initiative" was a massive failure - help stop it. http://bjoern.brembs.net/2016/o4/data-show-excellence-initiative-wasa-massive-failure-help-stop-it/ (letzter Zugriff am 13.5.2016).

Brembs, Björn / Brennicke, Axel (2015): Wir flexibilisieren uns zu Tode. Gegen das wachsende Wissenschaftsprekariat an den Universitäten gibt es ein einfaches Mittel: Verschlankt die Verwaltungen und gebt Wissenschaftlern feste Verträge! In: FAZ, 7.1.2015. http://www. faz.net/aktuell/feuilleton/forschung-und-lehre/verbesserung-der-arbeitsbedingungenan-unis-13354907.html (letzter Zugriff am 13.5.2016).

Engels, Anita / Beaufaÿs, Sandra / Kegen, Nadine V. / Zuber, Stephanie (2015): Bestenauswahl und Ungleichheit. Eine soziologische Studie zu Wissenschaftlerinnen und Wissenschaftlern in der Exzellenzinitiative. Frankfurt am Main: Campus.

Funken, Christiane / Rogge, Jan-Christoph / Hörlin, Sinje (2015): Vertrackte Karrieren. Zum Wandel der Arbeitswelten in Wirtschaft und Wissenschaft. Frankfurt am Main: Campus.

Graf, Angela (2015): Die Wissenschaftselite Deutschlands. Sozialprofil und Werdegänge zwischen 1945 und 2013. Frankfurt am Main: Campus.

Hartmann, Michael (2002): Der Mythos von den Leistungseliten. Spitzkarrieren und soziale Herkunft in Wirtschaft, Politik, Justiz und Wissenschaft. Frankfurt am Main: Campus.

Hartmann, Michael (2005): Elitehochschulen: Die soziale Selektion ist entscheidend. In: Prokla 34/137-4, 535-549.

Hartmann, Michael (2006): Die Exzellenzinitiative. Ein Paradigmenwechsel in der deutschen Hochschulpolitik. In: Leviathan 34/4, 447-465.

Hartmann, Michael (2010): Die Exzellenzinitiative und ihre Folgen. In: Leviathan 38/3, 369-387.

Hartmann, Michael (2013): Die Exzellenzinitiative und die Hierarchisierung des deutschen Hochschulsystems. In: NachDenkSeiten, http://www.nachdenkseiten.de/wp-print. php?p=16967 (letzter Zugriff am 14.5.2016). 
Hartmann, Michael (2015): Die Exzellenzinitiative und die Hierarchisierung des deutschen Hochschulsystems. In: Hans-Peter Müller / Tilman Reitz (Hg.), Bildung und Klassenbildung. Kritische Perspektiven auf eine Leitinstitution der Gegenwart. Weinheim: Beltz/ Juventa, 208-230.

Hasenjürgen, Brigitte (1996): Soziale Macht im Wissenschaftsspiel. SozialwissenschaftlerInnen und FrauenforscherInnen an der Hochschule. Münster: Westfälisches Dampfboot.

IEKE [Internationale Expertenkommission Exzellenzinitiative] (2016): Internationale Expertenkommission zur Evaluation der Exzellenzinitiative. Endbericht. http:// www.gwk-bonn.de/fileadmin/Papers/Imboden-Bericht-2016.pdf (letzter Zugriff am 14.06.2016).

Karabel, Jerome (2005): The Chosen. The Hidden History of Admission and Exclusion at Harvard, Yale, and Princeton. Boston: Houghton Mifflin.

Konsortium Bundesbericht wissenschaftlicher Nachwuchs (2013): Bundesbericht zur Situation des wissenschaftlichen Nachwuchses 2013. Statistische Daten und Forschungsbefunde zu Promovierenden und Promovierten in Deutschland. Bielefeld: W. Bertelsmann.

Kühl, Stefan (2016): Exzellente Beantragung. Eine alternative Evaluation der Exzellenzinitiative. Working Paper 1/2016, Universität Bielefeld.

Lange-Vester, Andrea / Teiwes-Kügler, Christel (2013): Zwischen W3 und Hartz IV. Arbeitssituation und Perspektiven von wissenschaftlichen Mitarbeiterinnen und Mitarbeitern. Opladen: Verlag Barbara Budrich.

Maeße, Jens (2015): Eliteökonomen. Wissenschaft im Wandel der Gesellschaft. Wiesbaden: Springer VS.

Merton, Robert K. (1985): Der Matthäus-Effekt in der Wissenschaft. In: Ders., Entwicklung und Wandel von Forschungsinteressen. Aufsätze zur Wissenschaftssoziologie. Frankfurt am Main: Suhrkamp, 147-171.

Merton, Robert K. (2010): Der Matthäus-Effekt in der Wissenschaft, II. Kumulativer Vorteil und der Symbolismus des intellektuellen Eigentums. In: Berliner Journal für Soziologie 20/3, 285-308.

Mettler, Suzanne (2014): Degrees of Inequality. How the Politics of Higher Education Sabotaged the American Dream. New York: Basic Books.

Möller, Christina (2015): Herkunft zählt (fast) immer. Soziale Ungleichheiten unter Universitätsprofessorinnen und -professoren. Weinheim/Basel: Beltz/Juventa.

Möllers, Christoph (2009): Kein Grundrecht auf Exzellenzschutz. In: Jürgen Kaube (Hg.), Die Illusion der Exzellenz. Lebenslügen der Wissenschaftspolitik. Berlin: Wagenbach, 56-64.

Münch, Richard (2007a): Die akademische Elite. Zur sozialen Konstruktion wissenschaftlicher Exzellenz. Frankfurt am Main: Suhrkamp.

Münch, Richard (2007b): Die Konstruktion von Elite-Universitäten durch soziale Schließung. In: Hermann-Josef Blanke (Hg.), Bildung und Wissenschaft als Standortfaktoren. Tübingen: Mohr Siebeck, 111-142.

Rivera, Lauren (2015): Pedigree. How Elite Students Get Elite Jobs. Princeton/Oxford: Princeton University Press.

Rogge, Jan-Christoph (2015): The winner takes it all? Die Zukunftsperspektiven des wissenschaftlichen Mittelbaus auf dem akademischen Quasi-Markt. In: Kölner Zeitschrift für Soziologie und Sozialpsychologie 67/4, 685-707.

Schulze-Cleven, Tobias / Olsen, Jennifer (2016): Worlds of Higher Education Transformed? Towards Varieties of Academic Capitalism. In: Higher Education, special issue: The New Political Economy of Higher Education, im Erscheinen.

Schwindt, Jürgen-Paul (2013): Exzellenz. In: Unbedingte Universitäten (Hg.), BolognaBestiarium. Zürich/Berlin: diaphanes, 101-103.

Stiftung neue Verantwortung (2011): Wege aus der Exzellenzfalle. Vorschläge für eine aktive Hochschulpolitik. Policy Brief 04/2011. http://www.stiftung-nv.de/sites/ default/files/o42011_policy_brief_wege_aus_der_exzellenzfalle.pdf (letzter Zugriff am 14.5.2016).

Weiss, Felix / Schindler, Steffen / Gerth, Maria (2015): Hochschulrankings als Kriterium für neue soziale Ungleichheit. In: Zeitschrift für Soziologie 44/5, 366-382. 